\title{
The Electric Field Modulation by Hemisphere Damage Sites in Fused Silica Subsurface
}

\author{
Shaobo He, ${ }^{1,2}$ Liang Yang, ${ }^{1,2} \mathrm{Li} \mathrm{Li},{ }^{1}$ and Xiao-Tao $\mathrm{Zu}^{1}$ \\ ${ }^{1}$ School of Physical Electronics, University of Electronic Science and Technology of China, Chengdu 610054, China \\ ${ }^{2}$ Research Center of Laser Fusion, China Academy of Engineering Physics, Mianyang 629100, China
}

Correspondence should be addressed to Shaobo He; heshaobo@caep.cn

Received 12 March 2014; Accepted 5 May 2014; Published 11 June 2014

Academic Editor: Wanguo Zheng

Copyright (c) 2014 Shaobo He et al. This is an open access article distributed under the Creative Commons Attribution License, which permits unrestricted use, distribution, and reproduction in any medium, provided the original work is properly cited.

The effect of defect density on the electric field modulation to incident laser is investigated in this work. Based on the actual defect distribution in fused silica subsurface, the three-dimension grid model of defect sites is constructed firstly. Then, the threedimension finite-difference time-domain method is developed to solve the Maxwell equations. The electric field intensity in the vicinity of the defect sites located in front subsurface of fused silica is numerically calculated. The relationships between the maximal electric field intensity in fused silica and the geometry of the defect sites are given. The simulated results reveal that the modulation becomes more remarkable with the increase of defects density firstly and then decrease. Besides, the effect of the distribution mode of defects on modulation is discussed. Meanwhile, the possible physical mechanism is analyzed in detail.

\section{Introduction}

Laser damage of optics could be seen as occurring in two distinct steps. The first one concerns the damage occurrence due to the first optic irradiation: the initiation step. Afterward, damage sites are likely to grow with successive new shots: the growth step [1-3]. Historically, the studies of laser damage have been focused on the first step, namely, the origin and nature of the initial damage site. The diagnosis of site and the nature of the precursors to the formation of the site are discussed mainly. Surely, such information is useful but must be taken in context when considering the design, construction, and operation of a major laser facility. As laser damage cannot be inhibited totally, any laser damage on an optic may increase in severity by beam obscuration, intensity modulation, and light scatter. Some methods have been proposed to inhibit the growth of that laser damage, such as exposure of the $\mathrm{SiO}_{2}$ surface damage site to pulses from a $\mathrm{CO}_{2}$ laser, operating at $10.6 \mathrm{~nm}$ wavelength and sputtering the $\mathrm{SiO}_{2}$ surface damage site by some ion beam [4-6]. However, the efficacy of the methods is inconclusive. Both treatment of $\mathrm{CO}_{2}$ laser and sputtering of ion beam can smooth the damage site surface but can seldom fill any larger site. By analyzing theoretically, once the damage site appears at the surface of fused silica, it may cause electric field enhancement of incident laser. The subject of this work is to address the intensity modulation of damage sites by computing the electric field intensity around the damage sites.

As for the field enhancement caused by defects, there have been some reports. The intensity distribution of an initially plane light wave incident on planar and conical surface cracks is calculated numerically by Génin et al. using a wave propagation computer code [7]. Qiu and Wolfea calculated the electric field distribution around conical pits to determine the optimal mitigation geometry which will not generate secondary damage precursors [8]. Stolz discussed the impact of irradiation wavelength on light intensification of a deeply imbedded spherical inclusion at normal and oblique incidence [9]. However, few literatures pay attention to the role of damages sites density during modulation and the effect of damage sites distribution on the modulation. At the actual damage sites distribution at optics subsurface, damage sites usually crowd together. So we built a grids model of three defects to discuss the modulation to electric field at different damage sites density and the effect of damage sites distribution on the modulation.

In the present work, the three-dimensional (3D) grid models of hemisphere damage sites are constructed firstly. 
Maxwell equations are solved by the 3D finite-difference time-domain method. Then the electric field intensity distribution in the vicinity of the damage sites in front subsurface of fused silica is numerically simulated. The relationships between the maximal electric field intensity in fused silica and the distribution of the damage sites are discussed. This work aims to provide the theoretical direction about the laser induced damage during inertial confined fusion (ICF).

\section{Model and Method}

After treatment of $\mathrm{CO}_{2}$ laser and sputtering of ion beam, the edge of damage site may melt and the bottom of damage site may be filled with fused silica. The most common shape of damage site is hemispherical. For simplicity, we assume each damage site is hemisphere and has the radius $r$ [10]. We build a 3D grid model of the damage site. In the model, the surface of damage site can be described by the 3D equation

$$
\left(x-x_{0}\right)^{2}+\left(y-y_{0}\right)^{2}+\left(z-z_{0}\right)^{2}=r^{2},
$$

where xoy plane is parallel to the fused silica surface. $\left(x_{0}, y_{0}, z_{0}\right)$ is the centre of the hemisphere site. The difference in grid size is $\delta=\lambda / 12=29.25 \mathrm{~nm}$.

The electric field intensity distribution in the vicinity of the damage sites is calculated using the 3D finite-difference time-domain method (FDTD) by solving Maxwell's equations [11, 12]. This method has been used to simulate numerous wave propagation and electromagnetic scattering problems encountered in photolithography [7]. During the numerical calculation, the perfectly matched layer (PML) boundary condition is adopted [12]. The relative dielectric constant $\varepsilon_{r}$ of fused silica is 2.25 . The $351 \mathrm{~nm}$ laser with an amplitude $1.0 \mathrm{~V} / \mathrm{m}$ travels in the direction normal to the surface, namely, along the $z$-axis. TM wave is considered in this work. Other damage in the fused silica is ignored. It is assumed that the damage sites are on the rear surface and the damage sites are empty $\left(\varepsilon_{r}=1.0\right)$. In simulation, the size of the damage sites is about 10 percent of the actual one due to the limitation of present computer internal storage.

\section{Results and Discussion}

3.1. Electric Field Modulations. First, the light intensity profiles of the damage sites are plotted using a gray scale at two different planes: (a) plane $y=70 \delta$ and (b) plane $z=101 \delta$ (see Figure 2). The gray scale is adapted so as to facilitate the comparison between the light intensity modulated by the damage sites and the incident light intensity. In the simulations of Figure 2, the radiuses of the three damage sites are all $15 \delta$. The centers of the three damage sites are in a given circle with the radius $R$. All the calculations are performed for normal-incidence illumination. Figure 2(a) refers to the electric field distribution at the plane $y=70 \delta$ and (b) refers to the electric field distribution at the exit plane $z=101 \delta$. In
Figure 2(a), the light intensity profile clearly displays the path of the beam reflected by the damage sites, the standing wave generated by these reflections, and the high intensity spots resulting from constructive interference between these waves. The simulations confirm that electric field is enhanced due to the existence of damage sites. Figure 2(b) is the light intensity profile at the exit plane. It is confirmed that the light intensity is enhanced remarkably at the exit plane, and the peak value of light intensity reaches $2.2 \mathrm{~V} / \mathrm{m}$. Also, the higher intensity spots appear at the rim of the damage sites. This phenomenon is in great agreement with corresponding experimental result of [13].

In order to discuss the effect of the damage sites density on electric field enhancement, we give the evolution of the maximal electric field intensity with $R$ for three different planes (see Figure 3). It can be seen that the evolution trends of the three curves are similar. These curves all increase firstly and then decrease. As three damage sites appear together at the fused silica surface, the incident wave is reflected by each damage site firstly and then interference occurs between the reflection waves. With the increase of $R$, in other words, with the decrease of damage sites density, $E_{\max }$ reaches its peak value as the condition of constructive interference is satisfied. With the further increase of $R$, the probability of the interference occurring between the reflections waves of each damage site decreases. So $E_{\max }$ decreases rapidly.

Also it can be observed from Figure 3 that peak value of $E_{\max }$ at exit plane is the highest. This shows that the enhancement is the most remarkable at exit plane. It is identical with the results of Figure 2 and the corresponding experiment [13].

From the evolution trend of each curve in Figure 3, we can find that for every curve there is a given $R$ at which the maximum of electric field is strongest. For example, the $R$ is $30 \delta$ for the curve describing the evolution of $E_{\max }$ with $R$ at the exit plane and $R$ is $22 \delta$ for the curve describing the evolution at the plane $y=100 \delta$. Another thing that should be pointed out is that the defect density decreases with the increasing of $R$ for a certain surface area.

3.2. The Effect of Distribution Mode on Modulation. In this section, the role of the distribution modes of damage sites played during modulation to electric field is discussed. Figures 4(a), 4(c), and 4(e) show that the electric field distribution as the centre of three damage sites is in a line, while (b), (d), and (f) show that the electric field distribution as the centre of three damage sites is in a circle as shown by Figure 1 . The radiuses of all damage sites are $15 \delta$. The damage sites densities can be regarded as the same for two distribution modes in a given area $S=\pi(R+r)^{2}$. Figures $4(\mathrm{a})$ and $4(\mathrm{~b})$ refer to the electric field distribution of the same plane $z=40 \delta$. Figures $4(\mathrm{c})$ and $4(\mathrm{~d})$ refer to the electric field distribution in plane $z=80 \delta$. Figures $4(\mathrm{e})$ and $4(\mathrm{f})$ refer to the electric field distribution in plane $z=100 \delta$, which is the rear surface of fused silica.

From Figure 4, an interesting phenomenon can be found. In spite of the variation of the electric field distribution, 


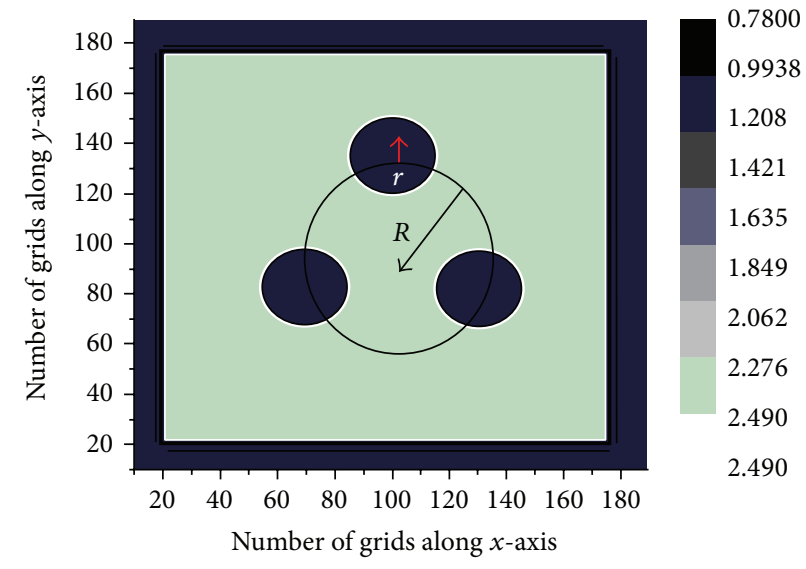

FIGURE 1: 2D schematic plots of damage sites.

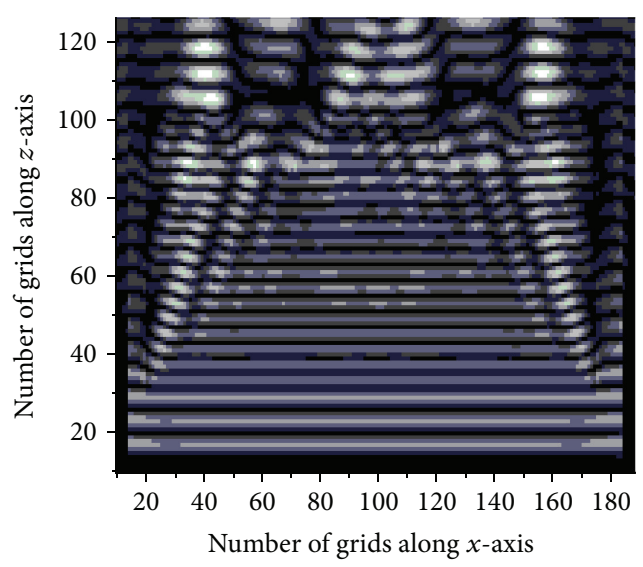

(a)

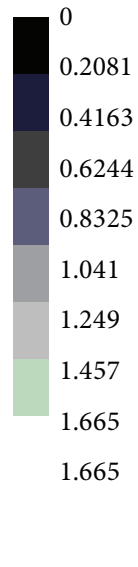

0
0.2081
0.4163
0.6244
0.8325
1.041
1.249
1.457
1.665
1.665

(1)

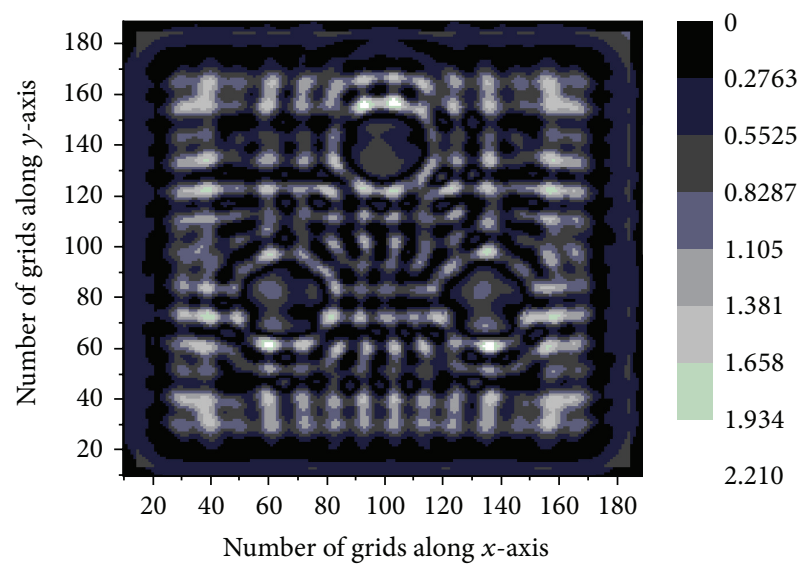

(b)

FIgURE 2: Distribution of electric field at (a) plane $y=70 \delta$ and (b) plane $z=101 \delta$.

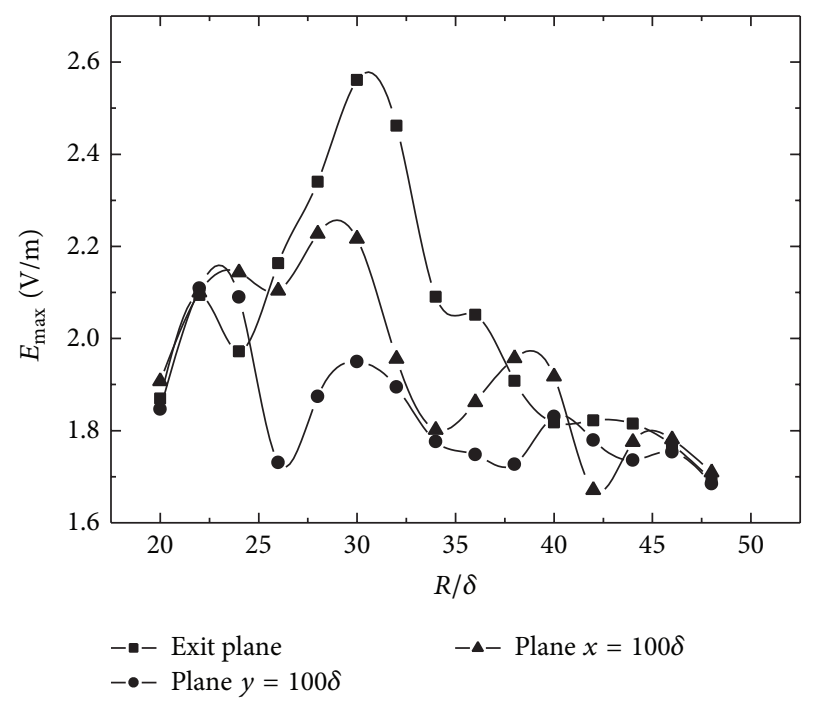

FIGURE 3: Plot of peak electric field intensity as a function of $R$. 


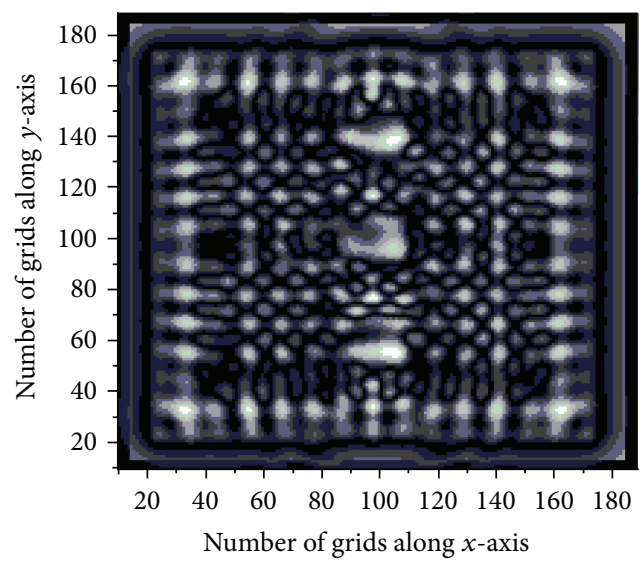

(a)

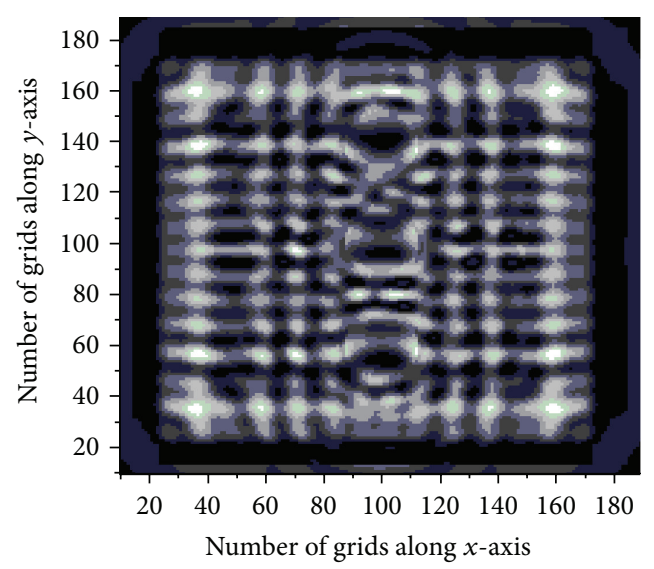

(c)

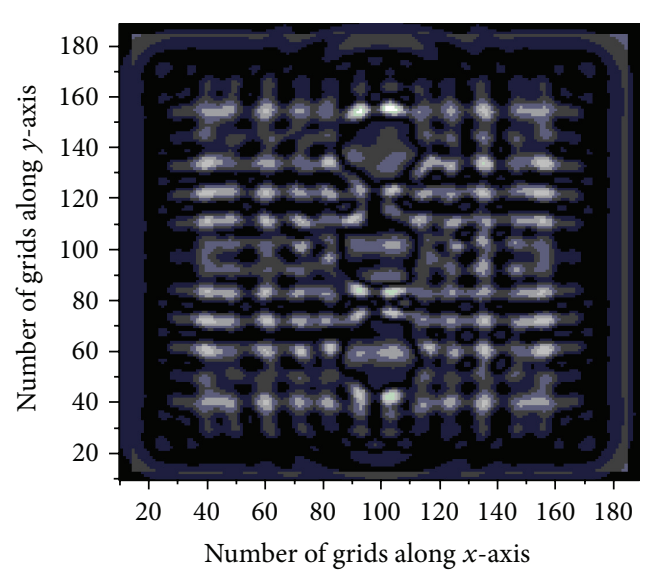

(e)
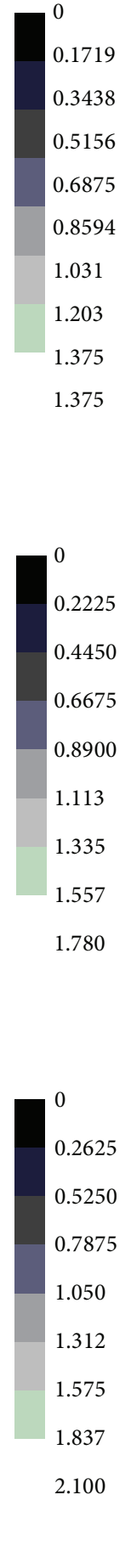

FIGURE 4: Electric field distribution for two kinds of damage site distribution mode.

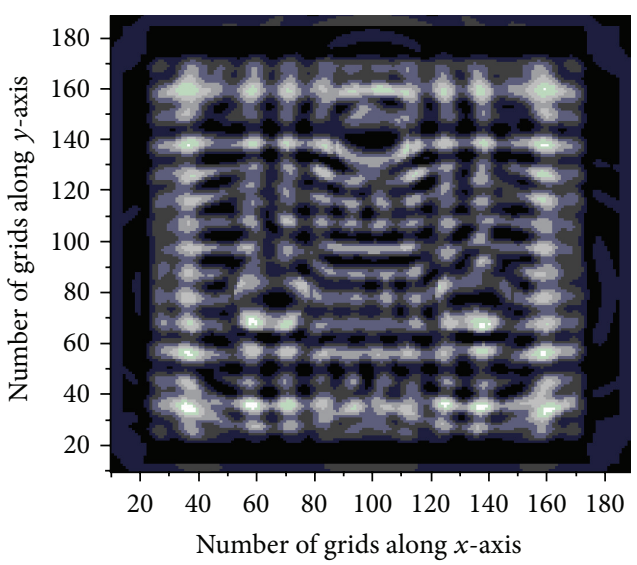

(d)

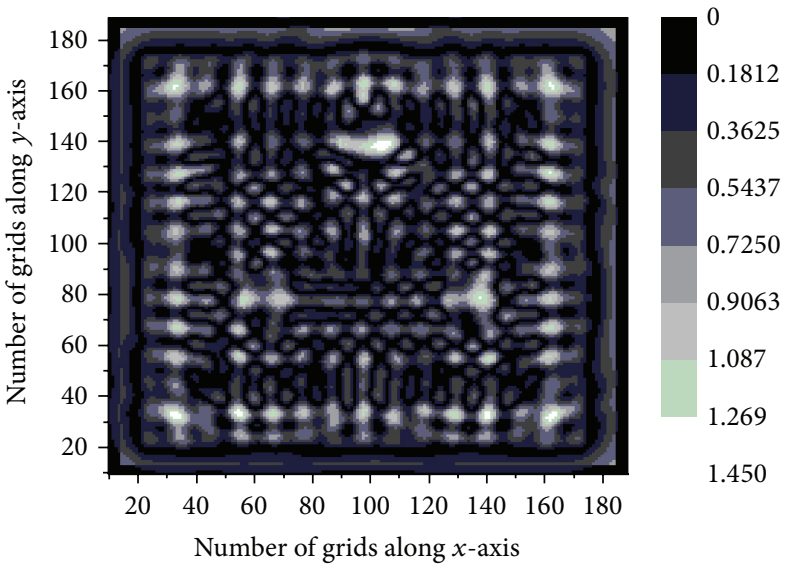

(b)

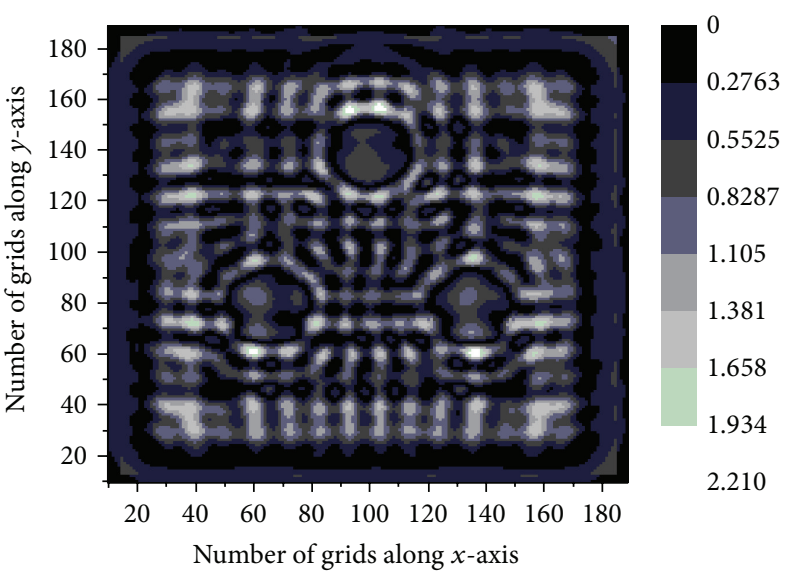

(f)

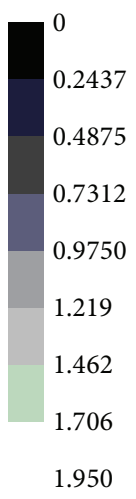

1.950 
TABLE 1: $E_{\max }(\mathrm{V} / \mathrm{m})$ for the two modes of three defects according to Figure 4.

\begin{tabular}{lccc}
\hline & $Z=40 \delta$ & $Z=80 \delta$ & $Z=100 \delta$ \\
\hline Distribution in a line & (a) 1.375 & (c) 1.700 & $(e) 2.130$ \\
Distribution in a circle & (b) 1.450 & (d) 1.950 & $(f) 2.210$ \\
Difference & 0.075 & 0.25 & 0.07 \\
\hline
\end{tabular}

obviously. With the decrease of damage sites density, a peak value of the maximum electric field appears. In the whole trend, the larger the damage sites density is, the stronger the modulation is.

(2) As the damage sites density is the same, the modulation is not too sensible to defect distribution mode.

\section{Conflict of Interests}

The authors declare that there is no conflict of interests regarding the publication of this paper.

\section{Acknowledgments}

This study was supported financially by the Fundamental Research Funds for the Central Universities (Grant no. ZYGX2010J045) and by the National Natural Science Foundation of China (61178018) and the Ph.D. Funding Support Program of Education Ministry of China (20110185110007).

\section{References}

[1] L. Lamaignère, G. Dupuy, T. Donval, P. Grua, and H. Bercegol, "Comparison of laser-induced surface damage density measurements with small and large beams: toward representativeness," Applied Optics, vol. 50, no. 4, pp. 441-446, 2011.

[2] M. D. Feit, A. M. Rubenchik, M. R. Kozlowski, F. Y. Genin, S. Schwartz, and L. M. Sheehan, "Extrapolation of damage test data to predict performance of large-area NIF optics at $355 \mathrm{~nm}$," in Laser-Induced Damage in Optical Materials, vol. 3578 of Proceedings of SPIE, pp. 226-234, Boulder, Colo, USA, September 1998.

[3] R. A. Negres, M. A. Norton, D. A. Cross, and C. W. Carr, "Growth behavior of laser-induced damage on fused silica optics under UV, ns laser irradiation," Optics Express, vol. 18, no. 19, pp. 19966-19976, 2010.

[4] R. M. Brusasco, B. M. Penetrante, J. A. Butler, and L. W. Hrubesh, "Localized $\mathrm{CO}_{2}$ laser treatment for mitigation of $351 \mathrm{~nm}$ damage growth on fused silica," in Laser-Induced Damage in Optical Materials, vol. 4679 of Proceedings of SPIE, pp. 40-47, Boulder, Colo, USA, October 2001.

[5] T. E. Felter, L. Hrubesh, A. Kubota, L. Davila, and M. Caturla, "Laser damage probability studies of fused silica modified by $\mathrm{MeV}$ ion implantation," Nuclear Instruments and Methods in Physics Research B: Beam Interactions with Materials and Atoms, vol. 207, no. 1, pp. 72-79, 2003.

[6] D. Wu, M. Lei, X. Zhu, and Y. Gong, "Numerical study on the ablation effects of tungsten irradiated by high-intensity pulsed ion beam," in Proceedings of the 1st International Conference on Physics Science and Technology (ICPST '11), pp. 246-251, December 2011.
[7] F. Y. Génin, A. Salleo, T. V. Pistor, and L. L. Chase, "Role of light intensification by cracks in optical breakdown on surfaces," The Journal of the Optical Society of America A, vol. 18, pp. 26072616, 2001.

[8] S. R. Qiu and J. E. Wolfea, "Modeling of light intensification by conical pits within multilayer high reflector coatings," in Proceedings of the Laser-Induced Damage in Optical Materials, vol. 7504 of Proceedings of the SPIE, December 2009.

[9] C. J. Stolz, "Light intensification modeling of coating inclusions irradiated at 351 and $1053 \mathrm{~nm}$," in Proceedings of the Conference Paper, Optical Interference Coatings (OIC '07), Laser Damage (FB), Tucson, Ariz, USA, June 2007.

[10] M. J. Matthews and M. D. Feit, 2007, SPIN, UCRL-PROC236247.

[11] D. B. Ge and Y. B. Yan, Electro-Magnetic Wave and FiniteDifference Fince-Dorucion, Xidian University Press, Xian, China, 2002.

[12] D. M. Sullivan, Electromagnetic Simulation Using the FDTD Method, IEEE Press, New York, NY, USA, 2000.

[13] L. Li, X. Xiang, and X. T. Zu, "Numerical simulation of the modulation to incident laser by the repaired damage site in a fused silica subsurface," Chinese Physics B, vol. 20, Article ID 074209, 2011. 

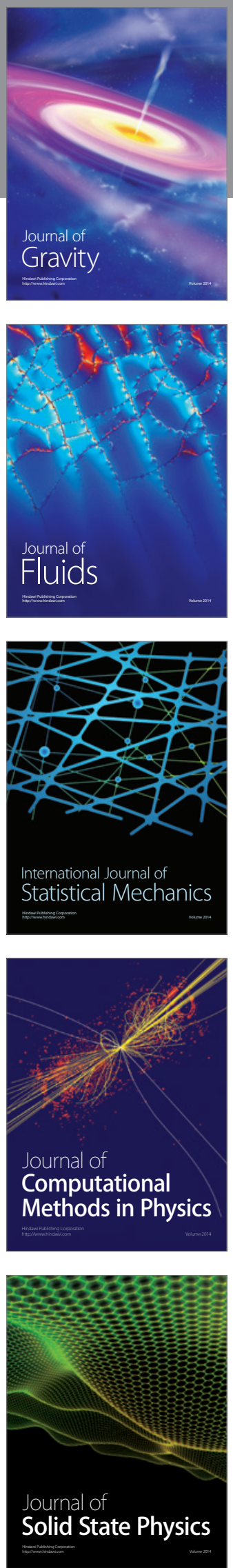

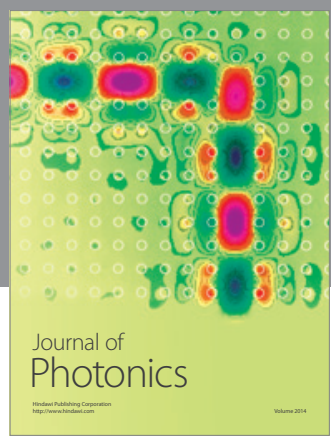

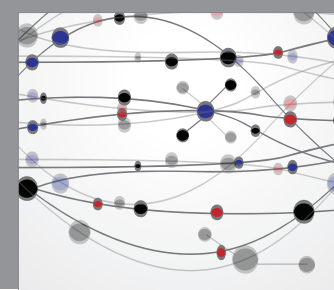

The Scientific World Journal

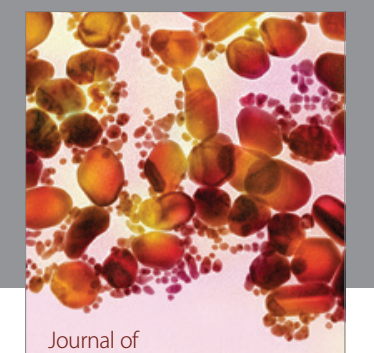

Soft Matter
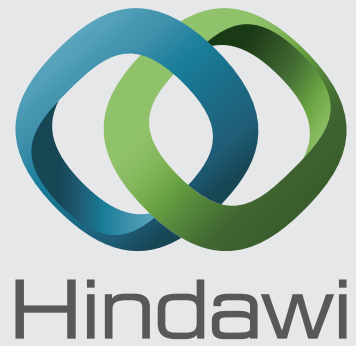

Submit your manuscripts at

http://www.hindawi.com
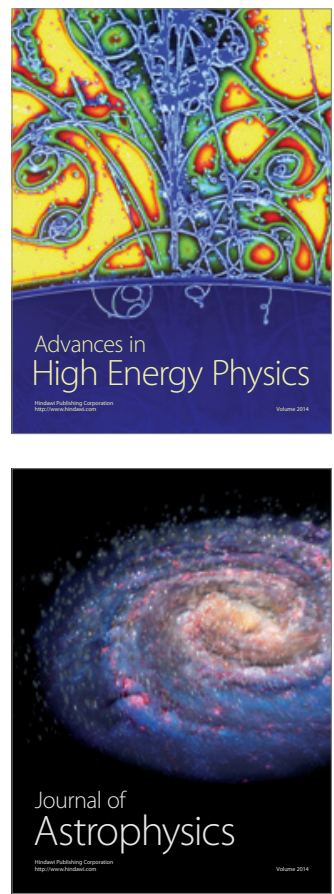
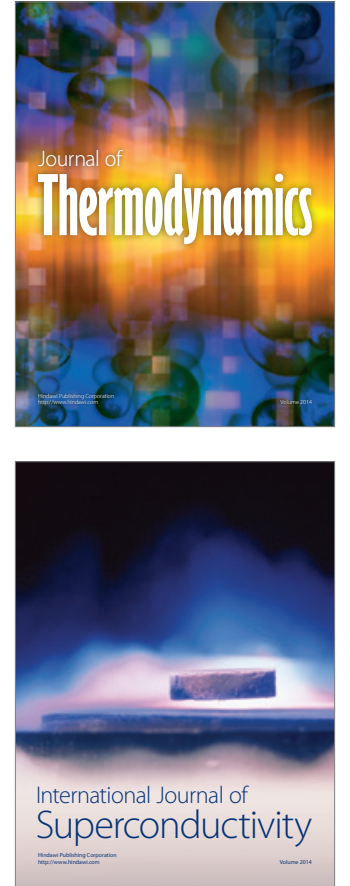
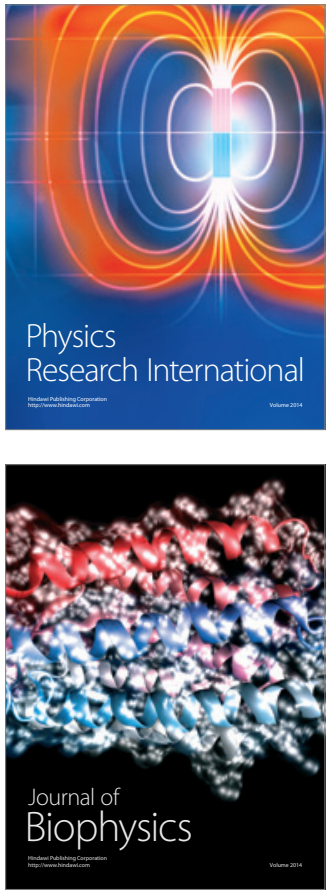
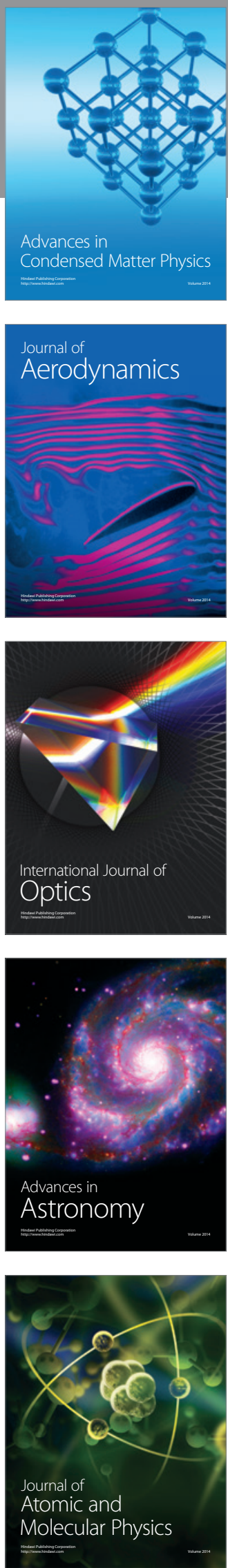